\title{
ZNF281 enhances cardiac reprogramming by modulating cardiac and inflammatory gene expression
}

\author{
Huanyu Zhou, ${ }_{1,2,3,7}$ Maria Gabriela Morales, ${ }^{1,2,3,7}$ Hisayuki Hashimoto, ${ }^{1,2,3}$ Matthew E. Dickson, ${ }^{1,2,3}$ \\ Kunhua Song, ${ }^{1,6}$ Wenduo Ye, ${ }^{1,2,3,8}$ Min S. Kim, ${ }^{4}$ Hanspeter Niederstrasser, ${ }^{5}$ Zhaoning Wang, ${ }^{1,2,3}$ \\ Beibei Chen, ${ }^{4}$ Bruce A. Posner, ${ }^{5}$ Rhonda Bassel-Duby, ${ }^{1,2,3}$ and Eric N. Olson ${ }^{1,2,3}$ \\ ${ }^{1}$ Department of Molecular Biology, University of Texas Southwestern Medical Center, Dallas, Texas 75390 , USA; ${ }^{2}$ Hamon Center \\ for Regenerative Science and Medicine, University of Texas Southwestern Medical Center, Dallas, Texas 75390 , USA; ${ }^{3}$ Senator \\ Paul D. Wellstone Muscular Dystrophy Cooperative Research Center, University of Texas Southwestern Medical Center, Dallas, \\ Texas 75390, USA; ${ }^{4}$ Department of Clinical Sciences, University of Texas Southwestern Medical Center, Dallas, Texas 75390, \\ USA; ${ }^{5}$ Department of Biochemistry, University of Texas Southwestern Medical Center, Dallas, Texas 75390, USA
}

Direct reprogramming of fibroblasts to cardiomyocytes represents a potential means of restoring cardiac function following myocardial injury. AKT1 in the presence of four cardiogenic transcription factors, GATA4, HAND2, MEF2C, and TBX5 (AGHMT), efficiently induces the cardiac gene program in mouse embryonic fibroblasts but not adult fibroblasts. To identify additional regulators of adult cardiac reprogramming, we performed an unbiased screen of transcription factors and cytokines for those that might enhance or suppress the cardiogenic activity of AGHMT in adult mouse fibroblasts. Among a collection of inducers and repressors of cardiac reprogramming, we discovered that the zinc finger transcription factor 281 (ZNF281) potently stimulates cardiac reprogramming by genome-wide association with GATA4 on cardiac enhancers. Concomitantly, ZNF281 suppresses expression of genes associated with inflammatory signaling, suggesting the antagonistic convergence of cardiac and inflammatory transcriptional programs. Consistent with an inhibitory influence of inflammatory pathways on cardiac reprogramming, blockade of these pathways with anti-inflammatory drugs or components of the nucleosome remodeling deacetylase (NuRD) complex, which associate with ZNF281, stimulates cardiac gene expression. We conclude that ZNF281 acts at a nexus of cardiac and inflammatory gene programs, which exert opposing influences on fibroblast to cardiac reprogramming.

[Keywords: direct cellular reprogramming; cardiomyocytes; ZFP281; cardiac gene activation; anti-inflammation; heart regeneration]

Supplemental material is available for this article.

Received August 2, 2017; revised version accepted September 7, 2017.

The adult heart is among the least regenerative tissues (Xin et al. 2013; Cahill et al. 2017). In response to myocardial infarction (MI), the human heart can lose hundreds of millions of cardiomyocytes, resulting in catastrophic loss of cardiac contractility. Typically, the lost cardiomyocytes are replaced by scar tissue due to activation of endogenous fibroblasts, which account for approximately a third of the cells of the heart (Tallquist and Molkentin 2017). Reprogramming cardiac fibroblasts (CFs) to induced cardiomyocytes (iCMs) by forced expression of cardiac transcription factors represents a potential means of

\footnotetext{
${ }^{6}$ Present address: Anschutz Medical Campus, University of Colorado, Aurora, CO 80045, USA.

${ }^{7}$ These authors contributed equally to this work.

${ }^{8}$ This work is dedicated to the memory of Wenduo Ye, an exceptional colleague and caring friend, who performed ChIP-seq experiments and bioinformatics analysis for this study.

Corresponding author: eric.olson@utsouthwestern.edu

Article is online at http://www.genesdev.org/cgi/doi/10.1101/gad.305482. 117.
}

enhancing cardiac repair by reducing scar tissue while simultaneously generating new cardiomyocytes (Ieda et al. 2010; Qian et al. 2012; Song et al. 2012; Fu et al. 2013; Nam et al. 2013). However, low efficiency as well as the lack of understanding of the molecular basis of the reprogramming process represent challenges to its potential clinical application (Srivastava and DeWitt 2016; Kojima and Ieda 2017; Vaseghi et al. 2017).

The first cardiac reprogramming cocktail consisted of three cardiac transcription factors: GATA4, MEF2C, and TBX5 (GMT) (Ieda et al. 2010). Subsequent effort has been directed toward optimization of cardiac reprogramming by generating different cocktails that contain various

(C) 2017 Zhou et al. This article is distributed exclusively by Cold Spring Harbor Laboratory Press for the first six months after the full-issue publication date (see http://genesdev.cshlp.org/site/misc/terms.xhtml). After six months, it is available under a Creative Commons License (Attribution-NonCommercial 4.0 International), as described at http://creativecommons.org/licenses/by-nc/4.0/. 
combinations of proteins, microRNAs, and small molecules (Song et al. 2012; Ifkovits et al. 2014; Muraoka et al. 2014; Wang et al. 2015; Yamakawa et al. 2015; Zhao et al. 2015; Zhou et al. 2015, 2016; Abad et al. 2017; Mohamed et al. 2017). Most of these studies adopted a candidate approach to examine the effects of supplementing the GMT cocktail with other key cardiac factors or regulators that have been shown to promote other somatic cell reprogramming processes. For example, adding the cardiac transcription factor HAND2, which plays an essential role in cardiac morphogenesis (Srivastava et al. 1997; Han et al. 2006; Firulli et al. 2017), enhanced the cardiac reprogramming efficiency of GMT both in vitro and in vivo (Song et al. 2012). Addition of a cardiac microRNA, miR-133, was also shown to enhance cardiac reprogramming in mouse and human fibroblasts (Nam et al. 2013; Muraoka et al. 2014). In addition, SB431542 (a TGF- $\beta$ inhibitor), and DAPT (a Notch inhibitor), which promote the reprogramming of induced pluripotent stem cells (iPSCs), also enhanced cardiac reprogramming (Ifkovits et al. 2014; Abad et al. 2017). However, these reprogramming cocktails are still relatively inefficient, and the molecular basis of the reprogramming process is poorly understood. Reprogramming of adult fibroblasts is also much less efficient compared with embryonic fibroblasts, posing challenges for potential restoration of adult cardiac function.

Previously, we screened a library of protein kinases and discovered that AKT1 dramatically accelerates and amplifies the cardiac reprogramming process (Zhou et al. 2015). Our optimal cocktail, which contains AKT1, GATA4, HAND2, MEF2C, and TBX5 (which we refer to as AGHMT), converts $~ 50 \%$ of mouse embryonic fibroblasts (MEFs) to iCMs (Zhou et al. 2015). However, the efficiency of conversion of adult fibroblasts, such as adult tail-tip fibroblasts (TTFs) and CFs, is still low.

Given that most known regulators of cardiac development or iPSC reprogramming have been tested extensively in previous studies and that none was able to efficiently reprogram adult fibroblasts to iCMs, we sought to identify previously unidentified regulators of cardiac reprogramming. Here we describe our findings from an unbiased screen of 1052 ORF cDNAs encoding 786 transcription factors, epigenetic regulators, cytokines, and nuclear receptors to augment AGHMT-dependent cardiac reprogramming of adult TTFs. This screen led to the discovery of 49 activators and 129 inhibitors of cardiac reprogramming, many of which participate in various signaling pathways and biological processes, including the TGF- $\beta$ and Notch signaling pathways, which have been shown to be important for cardiac reprogramming (Ifkovits et al. 2014; Abad et al. 2017). Interestingly, several factors involved in the inflammatory response were identified as inhibitors of cardiac reprogramming, indicating that the inflammatory response might act as a barrier for adult cardiac reprogramming. The strongest activator of cardiac reprogramming, Krüppel-type zinc finger transcription factor 281 (ZNF281), does not have a characterized function in cardiac development and thus would not have been anticipated to impinge on the mechanisms of fibroblast-to-cardiomyocyte reprogramming. We show that ZNF281 enhances cardiac reprogramming by associating with GATA4 on cardiac enhancers and inhibiting inflammatory signaling, which antagonizes cardiac reprogramming. Consistent with this notion, treatment of adult fibroblasts with anti-inflammatory drugs dramatically increased cardiac reprogramming efficiency. Our findings identify ZNF281 as a robust and efficient activator of adult cardiac reprogramming and highlight the importance of inflammatory signaling in the governance of cardiac gene expression.

\section{Results}

Identification of regulators of cardiac reprogramming in adult fibroblasts

Our previously optimized cardiac reprogramming protocol with five factors (AGHMT, also called $5 \mathrm{~F}$ ) was able to reprogram $\sim 3 \%$ of adult TTFs to iCMs as measured by activation of a cardiac-specific aMHC-GFP transgene and cardiac troponin $\mathrm{T}$ (cTnT) immunostaining (Zhou et al. 2015). To identify additional regulators of cardiac reprogramming, we created a retroviral expression library consisting of 1052 ORF cDNAs encoding 786 human transcription factors, cytokines, epigenetic regulators, and nuclear receptors (Supplemental Table S1). We initially screened this expression library for activators and inhibitors of cardiac reprogramming by expressing individual cDNAs together with $5 \mathrm{~F}$ in isolated TTFs from aMHC-GFP mice, as schematized in Figure 1A. After 9 d, a high-throughput cell analyzer system was used to image and quantify cardiac reprogramming based on aMHC-GFP and cTnT expression. Activators were defined as genes that increased aMHC-GFP or $\mathrm{c} T n T$ expression with a $Z$-score $\geq 2$, whereas genes with $Z$-scores -2 or lower for these cardiac markers were defined as inhibitors. This screen revealed 49 potential activators and 129 potential inhibitors of cardiac reprogramming (Fig. 1B,C; Supplemental Table S2).

Among the 49 activators, 25 enhanced aMHC-GFP expression, 35 enhanced cTnT expression, and 11 enhanced expression of both cardiac markers (Fig. 1B). The two strongest activators were PHD finger protein 7 (PHF7), a histone H3-binding protein expressed only in the male germline (Yang et al. 2012), and the ZNF281 protein, about which little is known (Fig. 1D; Supplemental Table S2). Among the 129 inhibitors, 121 inhibited aMHC-GFP expression, 41 inhibited cTnT expression, and 33 inhibited both cardiac markers (Fig. 1C). Some of the repressors, such as forkhead box protein A3 (FOXA3), nearly abolished 5F-mediated cardiac reprogramming (Fig. 1D; Supplemental Table S2). Cell numbers were unaffected by the inhibitors, suggesting that they acted directly on the reprogramming process rather than through indirect mechanisms, such as causing cell death.

\section{Pathway analysis of regulators of cardiac reprogramming}

To identify key pathways that regulate cardiac reprogramming, we performed pathway enrichment analysis for activator and inhibitor genes. Given that this was a genome- 
A

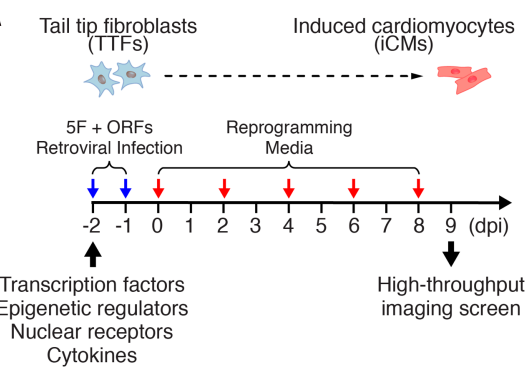

B

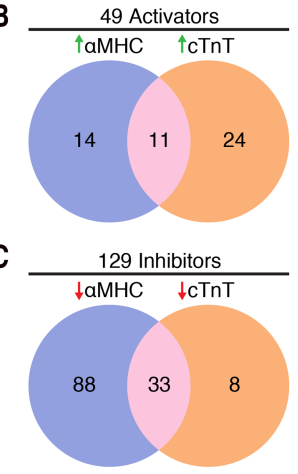

D

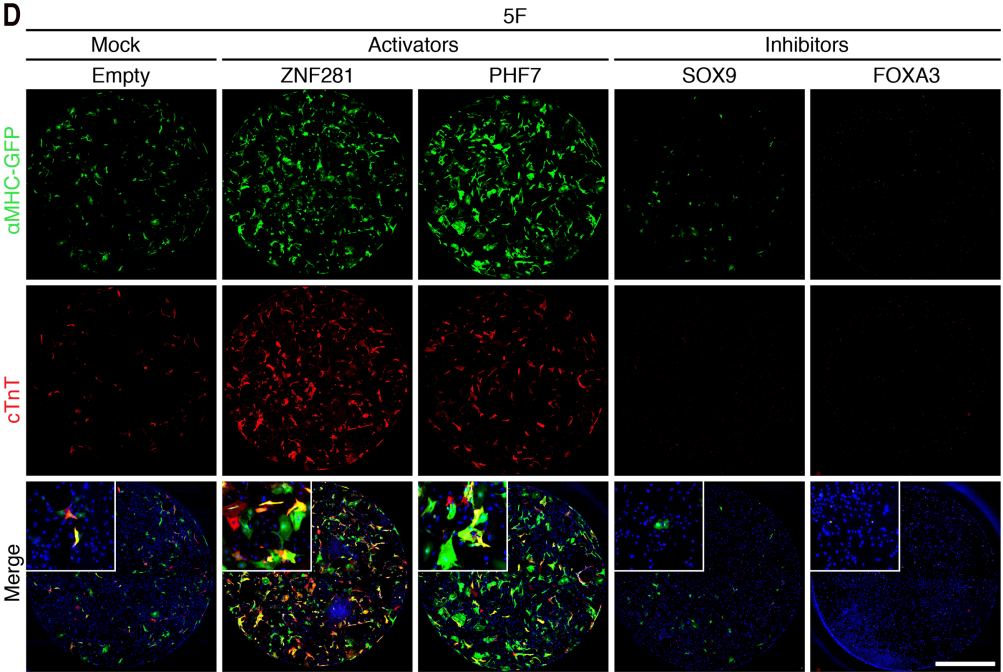

E

\begin{tabular}{|c|c|c|}
\hline & Pathway & Example \\
\hline \multirow{5}{*}{ Activators } & PI3K-Akt signaling pathway & ATF4, TP53, KITLG, SPP1, ATF2 \\
\hline & Anti-Inflammatory response & IFNA2, IFNA16, IL10 \\
\hline & cGMP-PKG signaling pathway & MEF2D, ATF4, GTF2IRD1, ATF2 \\
\hline & Cell cycle & TP53, SMAD2, TFDP1 \\
\hline & MAPK signaling pathway & MAX, ATF4, TP53, ATF2 \\
\hline \multirow{6}{*}{ Inhibitors } & Pro-Inflammatory response & $\begin{array}{l}\text { JUN, SMAD3, IL1A, IL2,CEBPB, TNFSF4, CXCL5, } \\
\text { CXCL13, ETS1, SMAD3, IL26, FASLG, CX3CL1 }\end{array}$ \\
\hline & TGF- $\beta$ s signaling pathway & BMP2, SMAD3, TGIF1, TGIF2, PITX2,SNAI2 \\
\hline & Notch signaling pathway & $\begin{array}{l}\text { HNF1B, ATOH1, TBX2, HOXD3, FOXA1, NR0B2, } \\
\text { SNAI2, SOX9 }\end{array}$ \\
\hline & $\begin{array}{l}\text { Signaling pathways regulating } \\
\text { pluripotency of stem cells }\end{array}$ & HNF1A, TBX3, OTX1, PAX6, SMAD3, ZIC3 \\
\hline & Osteoclast differentiation & FOS, JUN, MITF, PPARG, FOSL1, IL1A \\
\hline & Transcriptional misregulation in cancer & $\begin{array}{l}\text { ERG, CEBPB, CEBPE, PPARG, PAX5, TLX3, } \\
\text { FEV, KLF3 }\end{array}$ \\
\hline
\end{tabular}

Figure 1. Identification of activators and inhibitors of 5F-mediated cardiac reprogramming from a human ORF cDNA screen. (A) Schematic diagram of the human ORF cDNA library screen strategy for cardiac reprogramming in adult TTFs. (B) Venn diagram showing the number of activators identified from the screen. Genes with $Z$-scores of aMHC-GFP or cTnT expression $\geq 2$ were defined as activators. Twenty-five genes induced aMHC-GFP expression only, 35 genes induced cTnT expression only, and 11 genes induced expression of both markers. $(C)$ Venn diagram showing the number of inhibitors identified from the screen. Genes with Z-scores of aMHC-GFP or cTnT expression -2 or lower were defined as inhibitors. One-hundred-twenty-one genes repressed aMHC expression only, 41 genes repressed cTnT expression only, and 33 genes repressed both aMHC-GFP and cTnT expression. $(D)$ Representative immunocytochemistry images of TTFs from adult aMHC-GFP transgenic mice treated with $5 \mathrm{~F}$ and either empty virus or viruses encoding activators (ZNF281 or PHF7) and inhibitors (FOXA3 or SOX9). Cells were fixed and stained for aMHC-GFP (green), cTnT (red), and Hoechst (blue) 9 d after infection. Bars, $2 \mathrm{~mm}$. (E) Pathways enriched in activators $(n=$ $49)$ and inhibitors $(n=129)$, respectively, by DAVID pathway analysis. wide screen, we expected that this analysis would identify pathways known to regulate cardiac reprogramming. Indeed, the PI3K-AKT signaling pathway, which has been shown to enhance cardiac reprogramming (Zhou et al. 2015), was among the most enriched pathways associated with the activators. Other enriched pathways associated with the activators were the anti-inflammatory pathway, the cGMP-PKG signaling pathway, the cell cycle pathway, and the MAPK signaling pathway (Fig. 1E). It is noteworthy that the TGF- $\beta$ and Notch signaling pathways, which negatively regulate cardiac reprogramming (Ifkovits et al. 2014; Abad et al. 2017), were associated with the inhibitors. Other pathways associated with the inhibitors were the proinflammatory pathway and signaling pathways regulating pluripotency of stem cells, osteoclast differentiation, and transcriptional misregulation in cancer (Fig. 1E).

Because inflammatory signaling pathways were associated with both activators and inhibitors, we examined the functions of each individual gene within these pathways. Interestingly, we found that most of the identified activators possessed anti-inflammatory functions, including several anti-inflammatory cytokines, such as IFNA2, IFNA16, and IL10. Consistent with these findings, most identified inhibitors were proinflammatory, including several proinflammatory cytokines, such as IL1A, IL2, and IL26, and the inflammatory response transcription factor CEBP $\beta$ (Fig. 1E). 


\section{ZNF281 enhances cardiac reprogramming of adult fibroblasts}

PHF7 and ZNF281 were the two strongest activators identified from our retroviral cDNA expression screen with $5 \mathrm{~F}$ (Fig. 1D; Supplemental Table S2). We focused our initial attention on ZNF281, which has a broad expression pattern with enriched expression in the heart (Supplemental Fig. S1), and explored the mechanistic basis of its cardiac-inducing activity. Previous reports described the influence of ZNF281 on pluripotency, stemness, and epithelialmesenchymal transition (EMT) (Hahn and Hermeking 2014). However, the potential involvement of ZNF281 in cardiac development has not been explored previously.

We refer to our reprogramming mix of 5F plus ZNF281 as $6 \mathrm{~F}$. We validated the results of our screen by assessing GFP and cTnT expression in aMHC-GFP TTFs following $5 \mathrm{~F}$ and $6 \mathrm{~F}$ reprogramming after $7 \mathrm{~d}$ (Fig. 2A). Flow cytometry showed that addition of ZNF281 to $5 \mathrm{~F}$ generated $\sim 33 \%$ aMHC-GFP ${ }^{+}, \sim 45 \% \mathrm{cTnT}^{+}$, and $\sim 28 \%$ aMHC$\mathrm{GFP}^{+} / \mathrm{cTnT}^{+}$TTFs after $7 \mathrm{~d}$ of reprogramming (Fig. 2B, C). This TTF reprogramming efficiency using $6 \mathrm{~F}$ is noteworthy when considering the relatively low statistical likelihood of each fibroblast taking up all five or six separate retroviruses encoding the reprogramming factors. We also examined the expression of cardiac and fibroblast transcripts by quantitative PCR (qPCR). Addition of ZNF281 to $5 \mathrm{~F}$ increased the expression of cardiac marker genes Myh6 and Actc1 by $~ 120$-fold and 20-fold, respectively, and decreased expression of fibroblast marker genes Col1a2 and Sox 9 by $\sim 30 \%$ and $\sim 60 \%$, respectively (Fig. 2D). We also validated some of these results using aMHC-GFP CFs. The addition of ZNF281 to 5F increased the expression of GFP and cTnT in aMHC-GFP CFs evaluated by immunocytochemistry after $7 \mathrm{~d}$ of reprogramming (Supplemental Fig. S2A). These data were also corroborated by FACS analysis. The addition of ZNF281 to $5 \mathrm{~F}$ generated $\sim 25 \%$ aMHC-GFP ${ }^{+}, \sim 45 \% \mathrm{cTnT}^{+}$, and $\sim 21 \%$ aMHC-GFP ${ }^{+} / \mathrm{cTnT}^{+}$iCMs (Supplemental Fig. S2B,C). Interestingly, ZNF281 in the presence of $5 \mathrm{~F}$ also induced a twofold increase in the number of beating cells after 4 wk of reprogramming (Supplemental Fig. S2D).

\section{Global reprogramming of the cardiac transcriptome by ZNF281}

To further define the influence of ZNF281 on the reprogramming process, we performed RNA sequencing (RNA-seq) using adult TTFs reprogrammed for $7 \mathrm{~d}$ with $5 \mathrm{~F}$ or $6 \mathrm{~F}$. Using a twofold cutoff and false discovery rate (FDR) $<0.01$ threshold for inclusion, we identified $\sim 1000$ up-regulated genes and $~ 500$ down-regulated genes in 6F-treated compared with 5F-treated TTFs (Fig. 3A-C). Gene ontology (GO) enrichment analysis revealed that ZNF281 selectively up-regulated genes associated with muscle contractility (Fig. 3B,D), suggesting that ZNF281 globally enhanced cardiac reprogramming. Interestingly, the top GO terms enriched in the genes that were downregulated by ZNF281 were all related to the inflammatory response (Fig. 3C,E). The gene set enrichment analysis
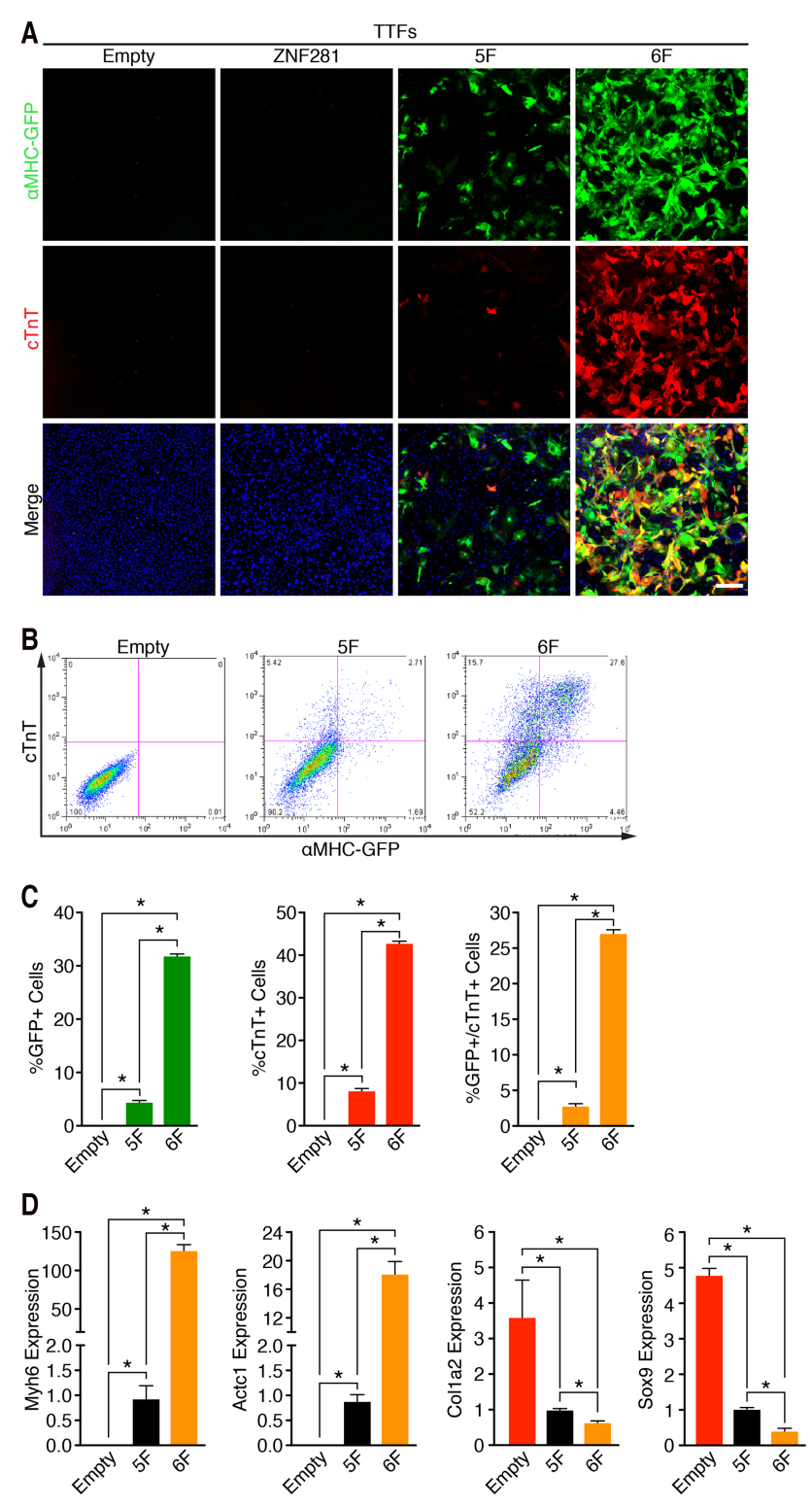

Figure 2. ZNF281 enhances cardiac reprogramming of adult fibroblasts. (A) Immunocytochemistry images of adult aMHCGFP transgenic TTFs $7 \mathrm{~d}$ after infection with empty, ZNF281, $5 \mathrm{~F}$, or $6 \mathrm{~F}$ retroviruses show that ZNF281 enhances expression of cardiac markers with 5F. (Green) aMHC-GFP; (red) cTnT; (blue) Hoechst. Bars, $500 \mu \mathrm{m} .(B, C)$ Representative flow cytometry plot $(B)$ and analyses $(C)$ of $\alpha M H C-G^{\prime} P^{+}$and $c \operatorname{TnT} T^{+}$TTFs $7 \mathrm{~d}$ after infection with empty, 5F, or 6F. (D) TTFs were infected with empty, 5F plus empty, and ZNF281 for $7 \mathrm{~d}$. Transcript levels of cardiac marker genes (Myh6 and Actc1) and fibroblast marker genes (Col1a2 and Sox9) were determined by qPCR. $\left({ }^{*}\right) P<0.05$.

(GSEA) also revealed that ZNF281 significantly up-regulated gene sets related to cardiac cells, such as myogenesis, muscle contraction, and heart processes (Fig. $3 \mathrm{~F}-\mathrm{H}$ ). Among the most up-regulated genes in these three gene sets, we found structural genes such as troponin $\mathrm{T}$ and troponin I isoforms (Tnnt1 and Tnnt3 Tnni3), the major cardiac muscle isoform of myosin heavy and light chain 
Zhou et al.

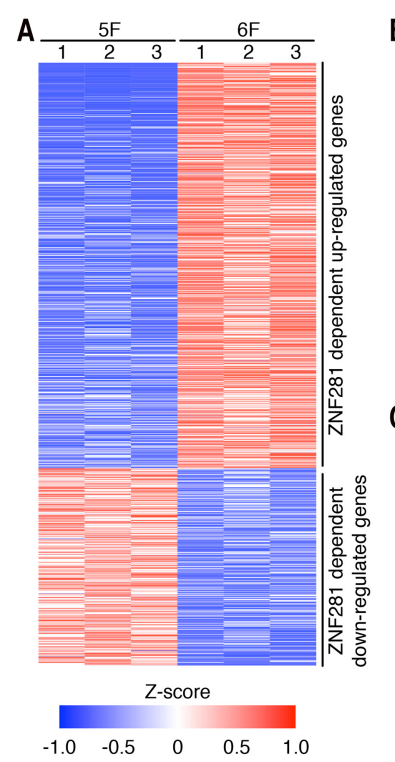

B
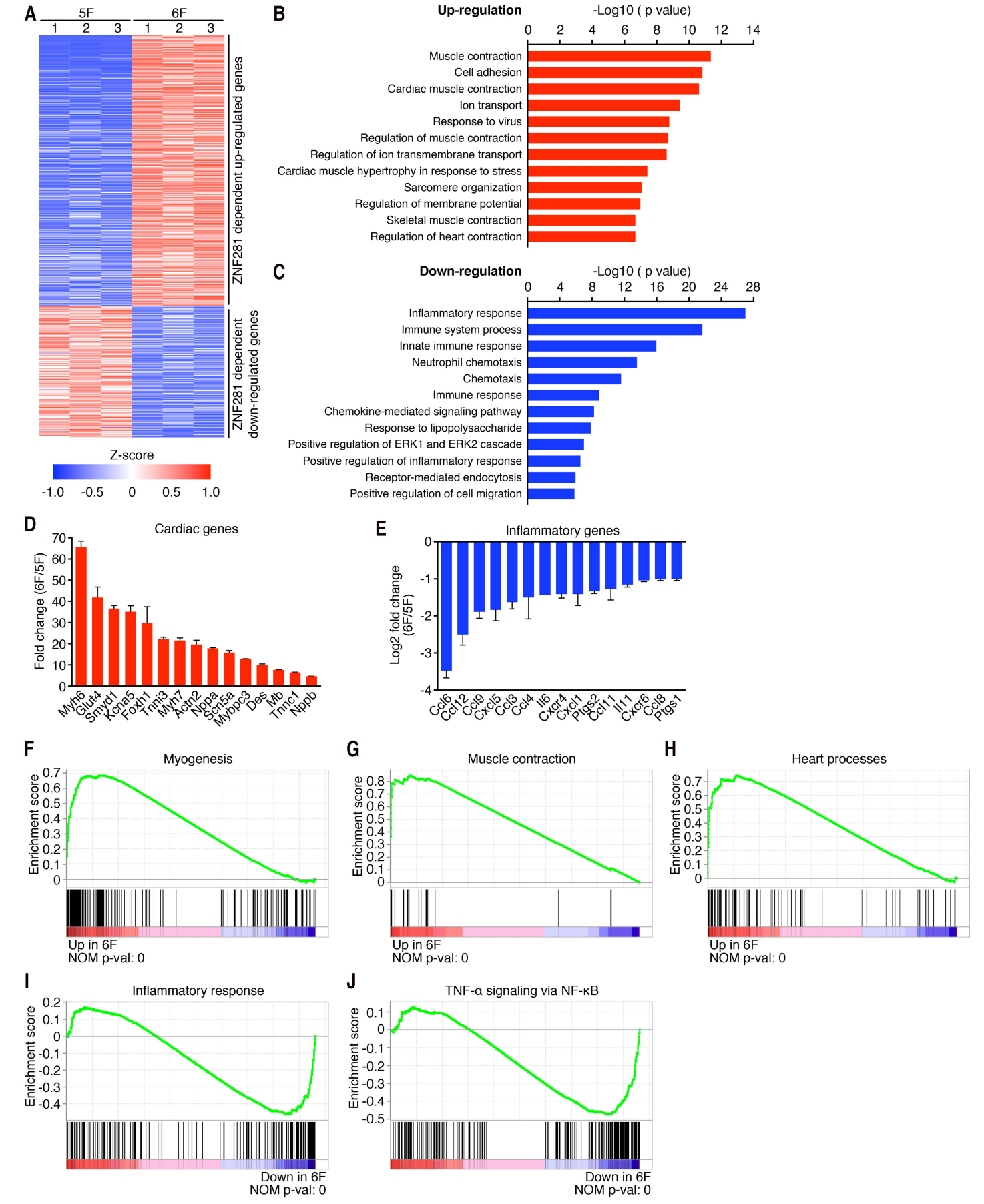

Figure 3. RNA-seq analysis shows that ZNF281 enhances cardiac genes and represses inflammatory genes. $(A)$ Heat map of 1500 differentially expressed genes in 5F-treated versus 6F-treated TTFs identified by RNA-seq. (Red) Up-regulation; (blue) down-regulation. RNAseq samples were prepared from adult TTFs reprogrammed for $7 \mathrm{~d} .(B, C) \mathrm{GO}$ analysis showing biological processes associated with genes up-regulated $(B)$ and down-regulated $(C)$ by ZNF281. $(D, E)$ Gene expression changes between $6 \mathrm{~F}$ and $5 \mathrm{~F}$ for selected cardiac markers $(D)$ or inflammatory markers $(E)$ as determined by RNA-seq. $(F-H)$ Enrichment plots of the indicated gene sets and their nominal $P$-value of genes up-regulated by ZNF281. $(I, J)$ Enrichment plots of the indicated gene sets and their nominal $P$-value of genes down-regulated by ZNF281.

(Myh6, Myh7, Myl4, and Myl2), specific muscle and cardiac actinin (Actc1 and Actn2), and the cardiac calcium channels (Ryr2), among other genes (Supplemental Fig. S3A-C). Similar to the GO analysis, ZNF281 in the presence of $5 \mathrm{~F}$ significantly down-regulated gene sets involved in the inflammatory response and TNF- $\alpha$ signaling via NF-kB. (Fig. 3I,J) The majority of the down-regulated genes by ZNF281 in all of these three gene sets encoded cytokines and cytokine receptors involved in the immune response (e.g., IL6, Marco, TNF-a, and IL7r) (Supplemental 
Fig. S3D,E). These results suggest a dual role for ZNF281 in the activation of cardiac and the suppression of inflammatory gene programs.

\section{ZNF281 broadly co-occupies cardiac enhancers with GATA4}

To begin to define the molecular mechanism by which ZNF281 enhances the cardiac reprogramming activity of the $5 \mathrm{~F}$ cocktail, we performed coimmunoprecipitation assays with ZNF281 and each reprogramming transcription factor in transfected HEK293 cells. We found that Flagtagged ZNF281 interacted with Myc-tagged GATA4 but not with HAND2, MEF2C, or TBX5 (Fig. 4A). To determine whether ZNF281 could directly activate cardiac genes, we examined the ability of ZNF281 to activate a luciferase reporter controlled by the aMHC promoter (aMHC-Luciferase). Indeed, ZNF281 activated the aMHC-Luciferase reporter approximately sevenfold, and when ZNF281 was coexpressed with GATA4, aMHC-Luciferase was activated $\sim 15$-fold (Supplemental Fig. S4), suggesting that ZNF281 and GATA4 cooperate to activate the aMHC promoter.

To further understand the molecular relationship between ZNF281 and GATA4 in the cardiac reprogramming process, we examined the genomic locations of ZNF281 and GATA4 at an early stage of the reprogramming process ( $2 \mathrm{~d}$ after infection with $6 \mathrm{~F}$ ) by chromatin immunoprecipitation (ChIP) with antibodies to endogenous proteins followed by high-throughput sequencing (ChIPseq) (Fig. 4B-E). De novo motif discovery on the binding peaks for ZNF281 and GATA4 revealed that the most significantly enriched motif associated with ZNF281 binding was GGGGTGGGG (Fig. 4C). For GATA4, the most enriched motif was GATAAG, which matches the consensus sequence for DNA binding of this transcription factor (Fig. 4D). ChIP-seq identified 14,623 peaks for ZNF281 and 30,664 peaks for GATA4 (Fig. 4E,F).

Based on the interaction of ZNF281 with GATA4, we predicted that ZNF281 co-occupies genomic sites with GATA4 on cardiac enhancers. Indeed, we found a high degree of overlap of ZNF281 and GATA4 genomic binding sites. Among the 14,623 peaks of ZNF281, we found
A

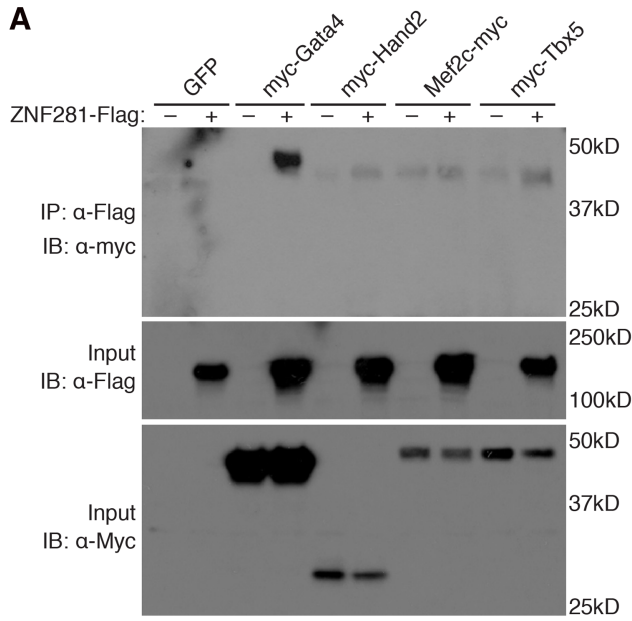

B

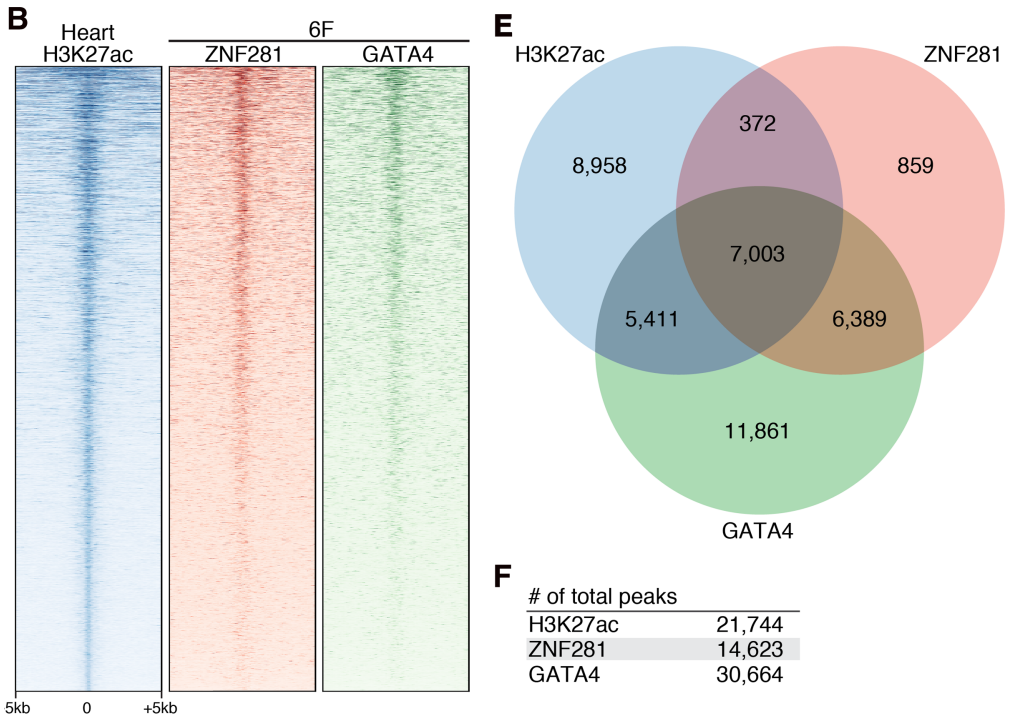

C ZNF281 binding motif

D

GATA4 binding motif

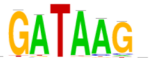

Figure 4. ZNF281 interacts with GATA4 to synergistically activate cardiac genes. $(A)$ Coimmunoprecipitation assays were performed using HEK293 cells transfected with equal amounts of plasmid DNA encoding Myc-tagged GATA4, HAND2, MEF2C, or TBX5 and/or Flag-tagged ZNF281. (IP) Immunoprecipitation; (IB) immunoblot. (B) Heat maps showing ChIP-seq (chromatin immunoprecipitation [ChIP] followed by high-throughput sequencing) data for H3K27ac in adult mouse hearts and ZNF281 and GATA4 binding in reprogrammed TTFs at $\pm 5 \mathrm{~kb}$ around the peak center. ChIP-seq experiments were performed using TTFs infected with $6 \mathrm{~F}$ for $2 \mathrm{~d} .(C)$ ZNF281-binding motif enriched within ZNF281binding peaks. $(D)$ GATA4-binding motif enriched within GATA4-binding peaks. (E) Venn diagram showing the number of overlapping peaks between heart H3K27ac, ZNF281, and GATA4. (F) Total number of peaks identified by ChIP-seq. 
that $91.6 \%(13,392$ of 14,623$)$ overlapped with peaks of GATA4 binding, and only $8.4 \%$ of ZNF281 peaks did not overlap with GATA4, indicating the co-occupancy of ZNF281 and GATA4 on endogenous genomic sites at an early stage of the cardiac reprogramming process (Fig. 4E). In contrast, the overlapping peaks of ZNF281 and GATA4 accounted for only $43.7 \%$ of the total GATA4 peaks $(13,392$ of 30,664$)$ (Fig. 4E), suggesting that GATA4 likely co-occupies additional sites with other reprogramming factors besides ZNF281.

To assess genome-wide localization of ZNF281 on cardiac enhancers, we established a chromatin landscape for heart tissue using H3K27ac ChIP data from The Mouse ENCODE Consortium (Mouse ENCODE Consortium et al. 2012) to reveal cardiac enhancers. Rank ordering of all cardiac enhancers based on $\mathrm{H} 3 \mathrm{~K} 27$ ac enrichment revealed that GATA4 bound to the majority $(57.1 \%$; $12,414$ of 21,744$)$ of active cardiac enhancers, consistent with the established role of GATA4 in heart development. ZNF281 bound to $33.9 \%$ (7375 of 21,744$)$ of active cardiac enhancers, while ZNF281 and GATA4 co-occupied 32.2\% (7003 of 21,744) of cardiac enhancers (Fig. 4B,E). Genomewide binding data for ZNF281 and GATA4 suggested that ZNF281 is most commonly associated with GATA4 on cardiac enhancers, defined by the presence of $\mathrm{H} 3 \mathrm{~K} 27 \mathrm{ac}$ (Fig. 4B,E).

\section{GATA4 recruits ZNF281 to cardiac enhancers}

We compared the occupancy patterns of ZNF281 in the presence (referred to as "6F") or absence of GATA4 (referred to as " $6 \mathrm{~F}-\mathrm{G}^{\prime}$ ) (Fig. 5A). Additionally, we examined the genomic occupancy pattern of GATA4 in the absence of ZNF281 (referred to as "6F - Z") following reprogramming (Fig. 5B). We found that the occupancy pattern of GATA4 throughout the genome was not dramatically affected by the presence or absence of ZNF281 (Fig. 5B). However, the presence of GATA4 strongly impacted the genomic occupancy pattern of ZNF281 (Fig. 5A). We assigned 33,934 ZNF281-binding peaks into three clusters based on how GATA4 influenced ZNF281 occupancy: a GATA4-dependent cluster (cluster 1, 8882 of 33,934 peaks), a GATA4-independent cluster (cluster 2, 7003 of 33,934 peaks), and a GATA4-inhibited cluster (cluster 3, 18,049 of 33,934 peaks) (Fig. 5A,B). Interestingly, cluster 3 , in which the occupancy of ZNF281 was inhibited by the presence of GATA4, also correlated with lower binding affinity of GATA4 compared with clusters 1 and 2 (Fig. 5A,B). It seems that the higher-affinity GATA4-binding sites in cluster 1 competed ZNF281 away from the lower-affinity GATA4-binding sites in cluster 3. For example, a region that is located at chromosome 1 $(137,694,960-137,749,970$; $\mathrm{mm} 9)$ shows binding peaks that belong to three different clusters (Fig. 5C). Binding peaks that associated with the sarcomere genes Tnnil and Tnnt2, which encode subunits of the troponin complex involved in regulating muscle contraction, were assigned to cluster 1 and cluster 2, respectively. Binding peaks that associated with a nonmuscle gene, ladinin-1
(Lad1), which encodes a basement membrane protein, were assigned to cluster 3 .

To investigate the functional significance of the GATA4- and ZNF281-binding peaks in the three clusters, we performed GO and pathway enrichment analysis using the Genomic Regions Enrichment of Annotations Tool (GREAT) (McLean et al. 2010). For GO enrichment analysis, we found that most of the GO terms enriched in clusters 1 and 2 were heart- or muscle-related (Fig. 5D), whereas most of the GO terms enriched in cluster 3 were related to stress and inflammatory responses. For pathway enrichment analysis, several pathways, such as the TGF- $\beta$ and Wnt signaling pathways, which are important for cardiogenesis, heart repair, and cardiac reprogramming (Ifkovits et al. 2014), were enriched in clusters 1 and 2 , whereas inflammatory response pathways were enriched in cluster 3 (Fig. 5E). We conclude that GATA4 directly recruits ZNF281 to cardiac enhancers to activate cardiac gene expression.

In contrast to the effect on cardiac enhancers, GATA4 did not attenuate ZNF281 binding to inflammatory enhancers (Fig. 5A,D,E). Additionally, ZNF281 served as a repressor instead of an activator of inflammatory genes, suggesting that ZNF281 acts through distinct mechanisms to regulate expression of cardiac and inflammatory genes.

Corepression of inflammatory genes by ZNF281 and nucleosome remodeling deacetylase (NuRD) complex components

Previously, it was reported that ZNF281 associates with the NuRD complex to repress transcription (Fidalgo et al. 2012, 2016). The NuRD complex is an ATP-dependent chromatin remodeling complex that contains multiple subunits. Notably, we identified the NuRD complex component MTA1 as an activator of cardiac reprogramming in our screen (Supplemental Table S2). Because the NuRD complex has also been shown to repress inflammatory signaling (Ramirez-Carrozzi et al. 2006), we tested other NuRD complex subunits, including MTA2, MTA3, MBD3, GATAD2A, GATAD2B, RBBP4, HDAC1, and HDAC2, in the reprogramming assay with $5 \mathrm{~F}$ in adult TTFs. Indeed, addition of four of the NuRD complex subunits (MTA1, MTA2, MTA3, and MBD3) to 5F substantially decreased expression of the inflammatory markers (IL6 and Ccl2) and increased expression of cardiac markers (Myh6 and Actc1) (Fig. 6A). Addition of NuRD complex subunits also promoted cardiac reprogramming, as measured by flow cytometry using the cardiac markers aMHC-GFP and cTnT (Fig. 6B; Supplemental Fig. S5A,B).

Because the NuRD complex is composed of several regulatory subunits, including HDAC1 and HDAC2, we tested an inhibitor of these enzymes (FK228) for its potential impact on reprogramming. Consistent with our model, we observed that inhibition of HDAC1/2 activity by FK228 decreased cardiac gene expression in the presence of $5 \mathrm{~F}$ to a level similar to control cells without 5F (Supplemental Fig. S5C,D). 


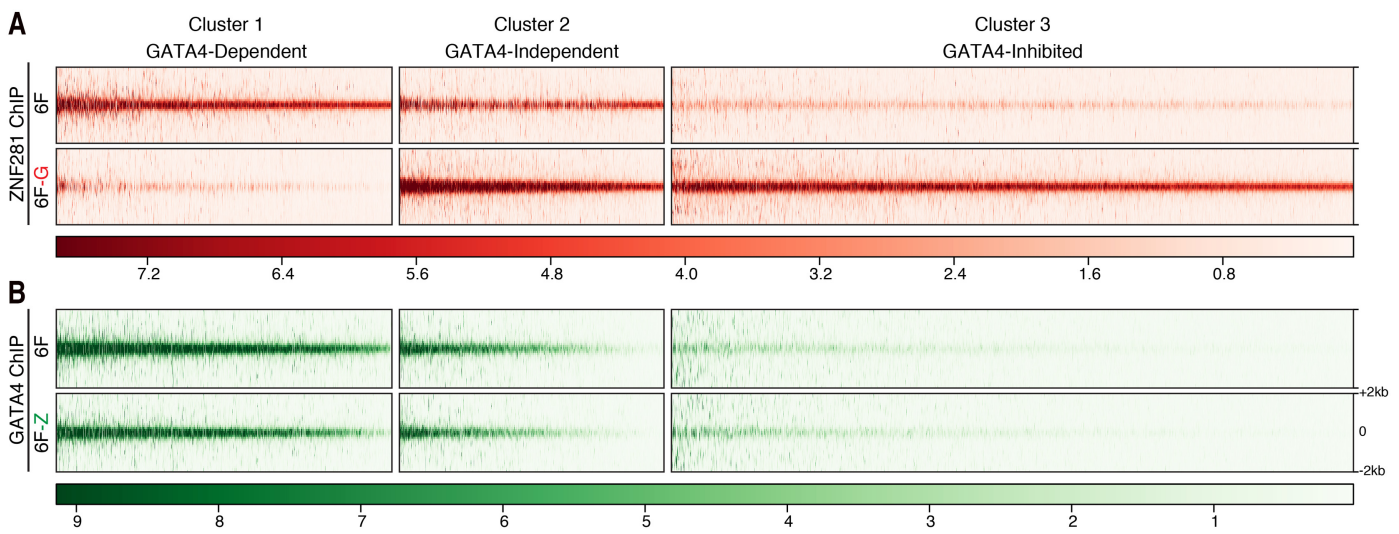

C

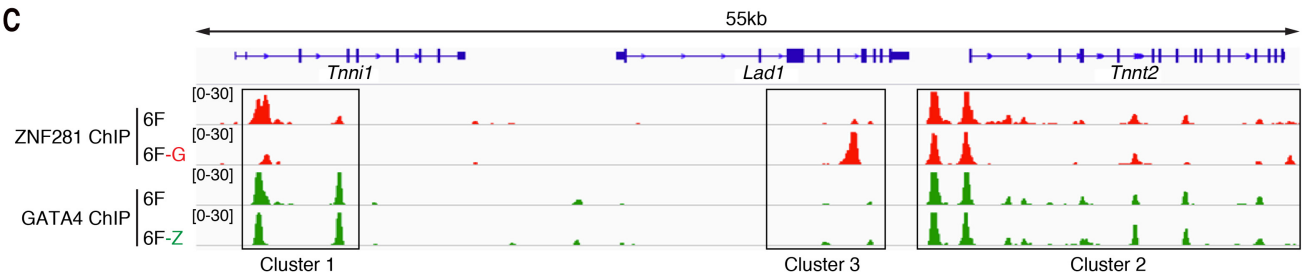

D
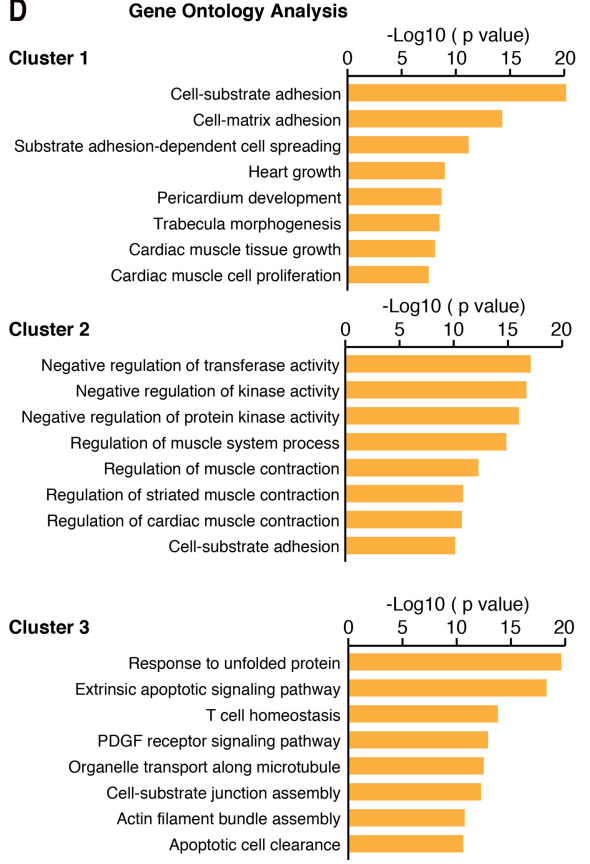

$E$
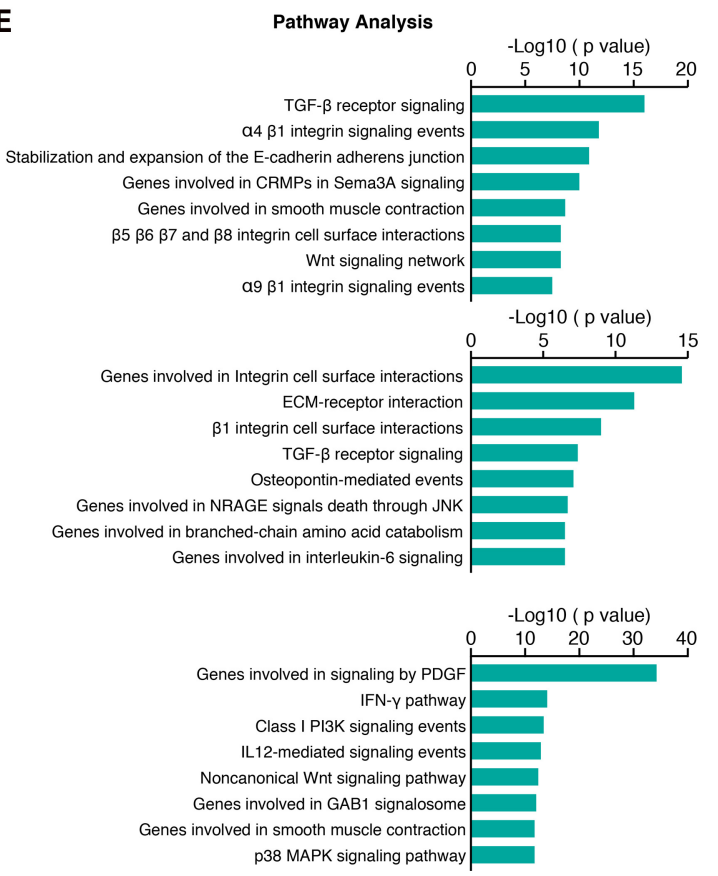

Figure 5. GATA4 recruits ZNF281 to cardiac enhancers. $(A, B)$ Heat map for ZNF281 or GATA4 genomic binding at $\pm 2 \mathrm{~kb}$ around the peak center in each cluster. ChIP-seq experiments were performed using adult TTFs reprogrammed for $2 \mathrm{~d}$ with $6 \mathrm{~F}, 6 \mathrm{~F}-\mathrm{G}$, or $6 \mathrm{~F}-\mathrm{Z}$. (C) Integrative Genomics Viewer (IGV) browser tracks at chromosome 1 (137,694,960-137,749,970; mm9) show an example of peaks that belong to each indicated cluster. $(D, E)$ GOs $(D)$ and pathways $(E)$ enriched in genes that associate with each indicated cluster identified by GO enrichment analysis and pathway analysis.

\section{Anti-inflammatory drugs promote cardiac reprogramming}

Given the influence of ZNF281 on inflammatory gene expression and the finding that numerous activators and inhibitors identified in our screen were also implicated in inflammatory pathways, we postulated that inhibition of the inflammatory response by anti-inflammatory drugs would also enhance reprogramming. To test this hypothesis, we evaluated the effects of two anti-inflammatory drugs-dexamethasone (Dex), a steroidal anti-inflammatory drug, and the cyclooxygenase enzyme inhibitor nabumetone ( $\mathrm{Nab}$ ), a nonsteroidal anti-inflammatory drug-on the reprogramming process. Anti-inflammatory drugs 

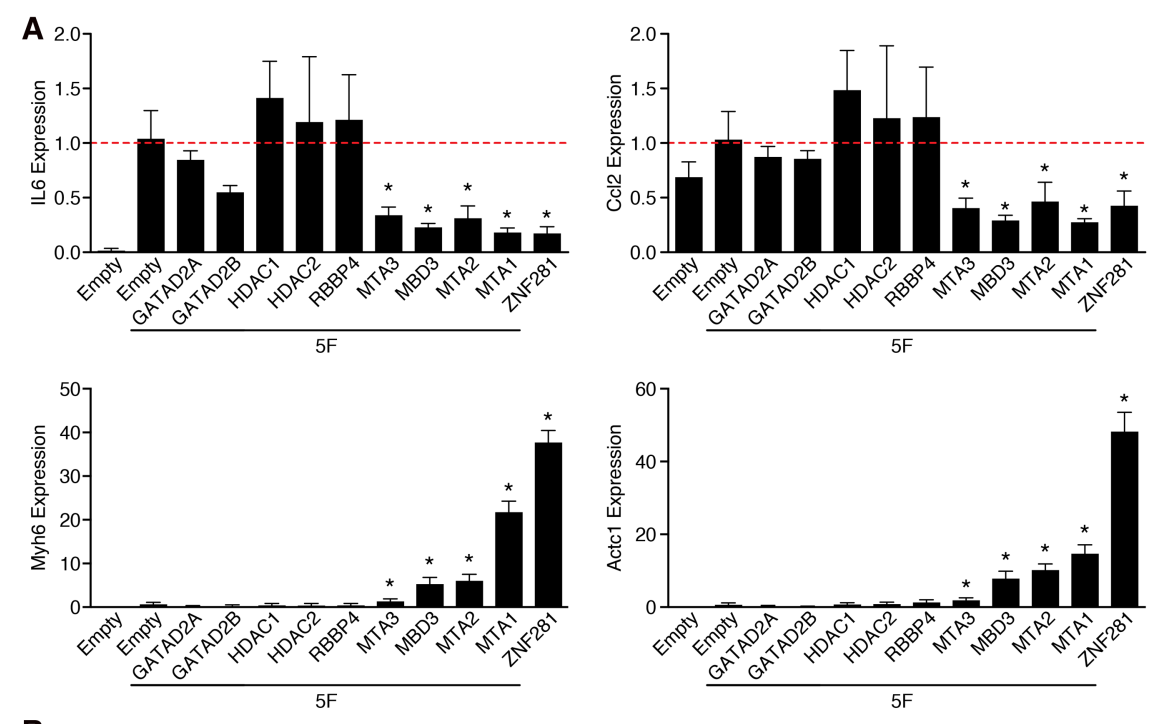

Figure 6. ZNF281 represses the inflammatory response through the NuRD complex. (A) TTFs were infected with empty, 5F plus empty, ZNF281, or each individual NuRD complex subunit retrovirus for 7 d. Transcript levels of inflammatory (IL6 and Ccl2) and cardiac (Myh6 and Actc1) marker genes were determined by qPCR. (B) Representative flow cytometry plot of $\alpha_{M H C-G F P}^{+}$and $\mathrm{CTnT}^{+}$TTFs $7 \mathrm{~d}$ after infection with empty, 5F plus empty, ZNF281, or each individual NuRD complex subunit retroviruses. The dashed line indicates control. $\left({ }^{*}\right) P<0.05$.

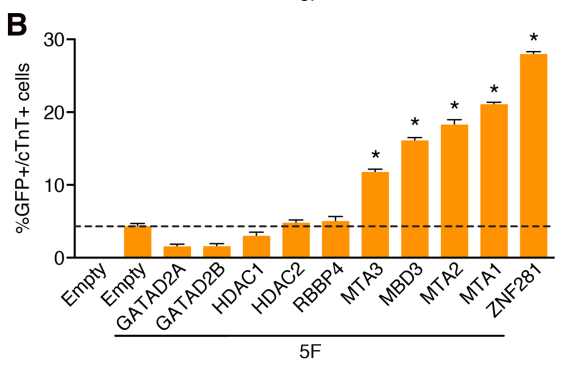

were added to 5F reprogrammed TTFs after viral infection. After $7 \mathrm{~d}$ of drug treatment, the transcript levels of inflammatory (IL6, Ccl2, and Ptgs1) and cardiac (Myh6, Actc1, and Nppa) markers were examined by qPCR. Addition of anti-inflammatory drugs to $5 \mathrm{~F}$ reprogrammed TTFs decreased expression of inflammatory markers, as expected, but increased the expression of cardiac markers from twofold to 10-fold, indicating enhanced reprogramming efficiency (Fig. 7A). Anti-inflammatory drugs also enhanced the reprogramming process as assayed by immunocytochemistry (Fig. 7B) and flow cytometry (Fig. 7C,D) for the cardiac markers aMHC-GFP and cTnT, respectively. Thus, we conclude that inflammatory signaling imposes an inhibitory influence on the cardiac reprogramming process.

\section{Discussion}

Here we performed an unbiased screen for regulators of adult cardiac reprogramming and identified 178 new activators and inhibitors that belong to various biological pathways. These different regulators revealed that antiinflammatory and proinflammatory factors evoke opposing effects on cardiac reprogramming. We found that proinflammatory molecules prevented reprogramming, whereas anti-inflammatory drugs enhanced cardiac reprogramming. Among the identified activators, ZNF281 showed the most potent stimulatory activity. The effect of ZNF281 on cardiac reprogramming appears to be mediated by association with GATA4 on cardiac enhancers and by inhibition of inflammatory signaling, which antagonizes cardiac reprogramming (Fig. 7E). We conclude that ZNF281 acts at the nexus of the opposing transcriptional programs for cardiac and inflammatory gene expression.

\section{Stimulation of cardiac reprogramming by ZNF281}

The potential involvement of ZNF281 in cardiac gene regulation has not been recognized previously. Our results indicate that ZNF281 functions as a positive regulator of cardiogenesis by associating with GATA4 on cardiac enhancers, resulting in synergistic activation of a broad collection of cardiac genes, including those encoding cardiac transcription factors, calcium-handling proteins, cardiac metabolic enzymes, and components of the sarcomere. ZNF281 is expressed in a variety of tissues during various developmental stages, and its absence in mice results in embryonic lethality between embryonic days 7.5 and 8.5, prior to the formation of primitive ventricles and atria (Fidalgo et al. 2011; Xin et al. 2013). We speculate that ZNF281 may also have important functions in the early stage of cardiac development.

It has been suggested that reprogramming requires pioneer factors to first engage and open target sites in chromatin and confer competency for other factors to bind (Soufi et al. 2015). Previously, GATA4 was shown to serve as a pioneer factor for the albumin gene enhancer in liver precursor cells by initiating chromatin opening (Cirillo et al. 2002). Cardiac gene expression during heart development requires regulated interactions among the 
A

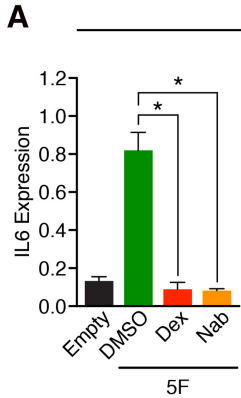

B
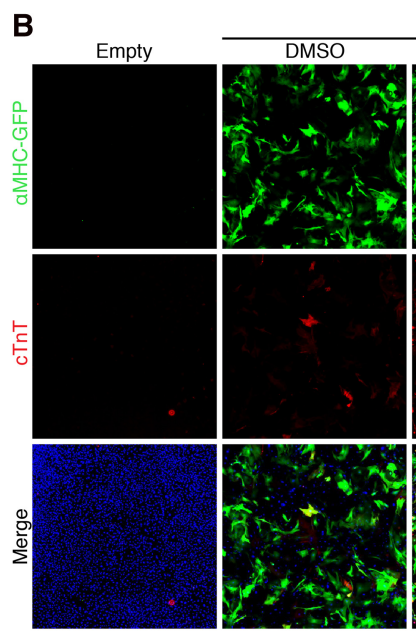

$5 \mathrm{~F}$
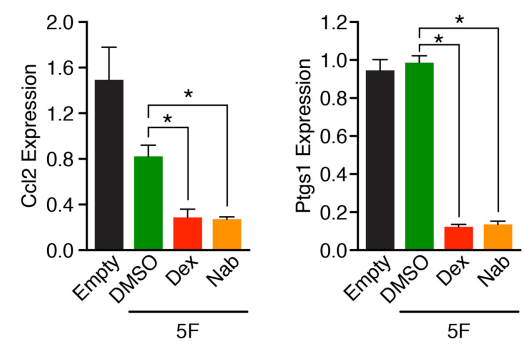

sec
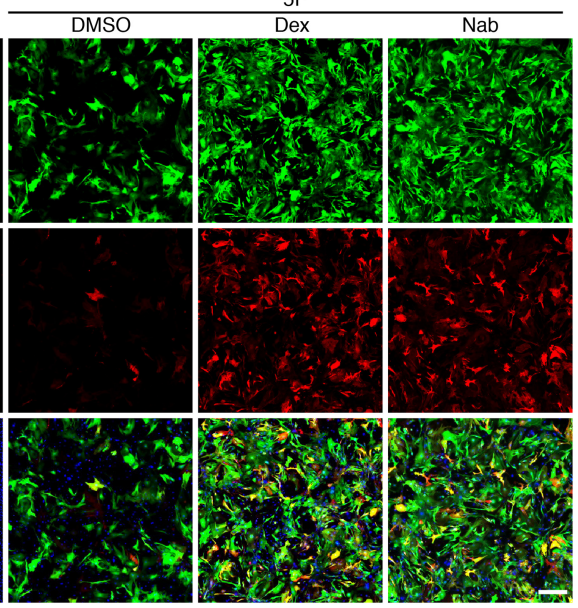

E

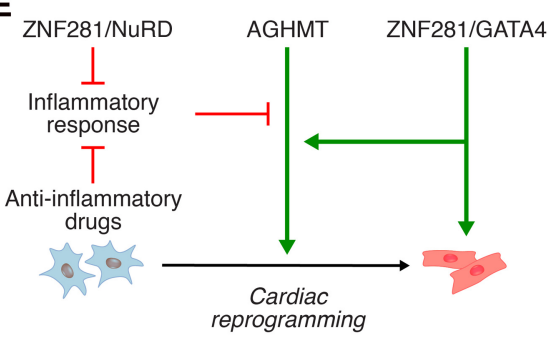

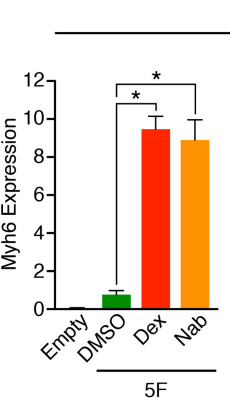

Cardiac genes

c
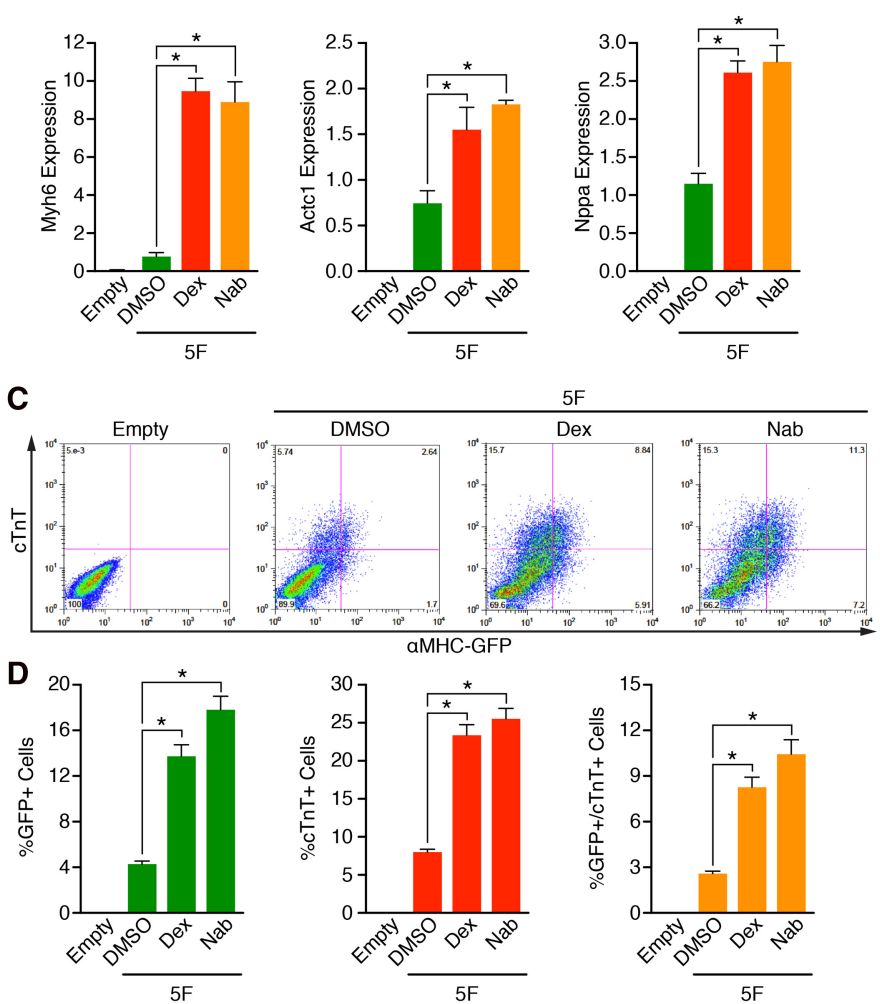

$5 \mathrm{~F}$

Figure 7. Anti-inflammatory drugs promote cardiac reprogramming. $(A) 5 \mathrm{~F}$ reprogrammed TTFs treated with either DMSO or the indicated anti-inflammatory drugs for $7 \mathrm{~d}$ after infection. Expression of inflammatory genes (IL6, Ccl2, and Ptgs1), and cardiac genes (Myh6, Actc1, and Nppa) was determined by qPCR. (Dex) $10 \mu \mathrm{M}$ Dex; (Nab) $10 \mu \mathrm{M} \mathrm{Nab}$. (B) Immunocytochemistry images of 5F reprogrammed adult aMHC-GFP transgenic TTFs treated with DMSO or the indicated anti-inflammatory drugs for $7 \mathrm{~d}$. (Green) aMHC-GFP; (red) cTnT; (blue) Hoechst. Bars, $500 \mu \mathrm{m}$. $(C, D)$ Representative flow cytometry plot $(C)$ and analyses $(D)$ of $\alpha \mathrm{MHC}^{-\mathrm{GFP}^{+}}$and cTnT ${ }^{+}$cells in $5 \mathrm{~F}$ reprogrammed adult aMHC-GFP transgenic TTFs treated with DMSO or the indicated anti-inflammatory drugs for $7 \mathrm{~d}$. $\left(^{*}\right) P<0.05$. $(E)$ Model showing the mechanism of action of ZNF281 on 5F-mediated direct cardiac reprogramming. ZNF281 is a cardiac transcription coactivator recruited by GATA4 to cardiac enhancers to activate cardiac gene expression. ZNF281 also represses the inflammatory response, which acts as a barrier pathway to cardiac reprogramming.

transcription factors GATA4, TBX5, and NKX2-5. The combined cooperativity of these factors is critical for the expansion of cardiac progenitors and the regulation of the cardiac differentiation program (Luna-Zurita et al. 2016). Moreover, it has been shown in human cardiomyocytes that GATA4 broadly co-occupies cardiac enhancers with TBX5 (Ang et al. 2016). This association is physiologically relevant because a human mutation in GATA4 that disrupts its association with TBX5 leads to aberrant chromatin states and impaired contractility, calcium handling, and metabolic activity (Ang et al. 2016).
We show that ZNF281 does not bind to cardiac enhancers in fibroblasts without the presence of GATA4, indicating that ZNF281 is unlikely to be a pioneer factor that opens chromatin. However, we show that ZNF281 requires the presence of the pioneer factor GATA4 to bind cardiac enhancers. Understanding the mechanism by which pioneer factors and other transcription factors interact and induce conformational changes in chromatin structure will be necessary to decipher the molecular mechanism involved in cardiac reprogramming. 


\section{The influence of inflammation and the NuRD complex} on cardiac reprogramming

Following injury, inflammatory infiltration is among the earliest responses, which is necessary to clear cellular debris and promote scar formation (Aurora and Olson 2014). Recent studies revealed both positive and negative roles for the inflammatory response in cardiac repair and regeneration. Inhibition of C/EBP signaling in the adult epicardium reduces injury-induced neutrophil influx, leading to a reduction of fibrosis and maintenance of cardiac function (Huang et al. 2012). In contrast, the injuryinduced macrophage response is necessary for heart regeneration in neonatal mice and zebrafish (Aurora et al. 2014; Lai et al. 2017). Our results show that the inflammatory response acts as a barrier for cardiac reprogramming, and ZNF281 appears to impose an anti-inflammatory influence on cardiac reprogramming.

The NuRD chromatin remodeling complex has been shown to repress proinflammatory gene expression in macrophages (Ramirez-Carrozzi et al. 2006). We show that in addition to direct cardiac gene activation, ZNF281 and some components of the NuRD complex also repress inflammatory signaling and activate cardiac reprogramming. Unlike cardiac reprogramming, ZNF281 has been shown to repress iPSC reprogramming through the NuRD complex (Fidalgo et al. 2011). Given that the inflammatory response is critical for iPSC formation (Lee et al. 2012), we surmise that in addition to repression of embryonic stem cell-specific genes, the anti-inflammatory effect of the ZNF281/NuRD complex also contributes to the repression of iPSC reprogramming. We cannot exclude the possibility that the NuRD complex has a more direct role in the activation of the cardiac gene program. In this regard, preservation of the identity of heart or skeletal muscle cell types has also been shown to depend on NuRD-mediated epigenetic repression of alternate lineage gene expression. Loss of the NuRD subunit Chd4 in the heart triggers aberrant expression of skeletal muscle gene expression and leads to hybrid striated muscle tissue (Gomez-Del Arco et al. 2016).

The opposing effects of inflammation on cardiac versus iPSC reprogramming suggest that these two types of reprogramming use distinct regulatory mechanisms. Given that embryonic stem cells are pluripotent and able to generate different cell types, whereas cardiomyocytes are terminally differentiated cells, it is possible that inflammation promotes epigenetic remodeling that is favorable for iPSC but not cardiac reprogramming.

\section{Clinical implications}

Direct reprogramming of adult fibroblasts to cardiomyocytes represents a potential approach for repair of the heart following injury. As an activator of cardiac reprogramming, ZNF281 offers new insights into the molecular basis of this process. Additionally, our finding that ZNF281 represses the inflammatory response reveals a role for anti-inflammatory signaling in cardiac reprogramming and highlights the potential clinical application of commonly used anti-inflammatory drugs in cardiac repair.

\section{Materials and methods \\ Isolation of adult mouse fibroblasts}

For isolation of adult mouse TTFs, tails were cut from 8- to 12wk-old adult wild-type or aMHC-GFP mice and minced into 1$\mathrm{cm}$ pieces with razor blades after peeling off the superficial dermis. The minced tails were placed in fibroblast growth medium (DMEM supplemented with 10\% FBS and 1\% [v/v] penicillin/ streptomycin). TTFs migrated out from the explants within 1 wk and were passaged once before use. For the adult mouse CF isolation, hearts from 8- to 12-wk-old adult wild-type or aMHCGFP mice were chopped with razor blades into small pieces. The chopped hearts were digested for $1 \mathrm{~h}$ at $37^{\circ} \mathrm{C}$ in a digestion medium containing Liberase TH and Liberase TM (Roche), DNase I (New England Biolabs), and polaxomer (Sigma). After digestion, cell suspensions were filtered through a $70-\mu \mathrm{m}$ strainer and placed in fibroblast growth medium (DMEM supplemented with $10 \%$ FBS and $1 \%$ [v/v] penicillin/streptomycin). All animal experiments described in this study were approved and conducted under the oversight of the University of Texas Southwestern Institutional Animal Care and Use Committee.

\section{Construction of the human retroviral ORF CDNA library}

Gateway-compatible human ORF pEntry vectors were purchased from Thermo Fisher Scientific. Gateway-compatible retroviral destination vector pMXs-gw was a gift from Shinya Yamanaka (Addgene, plasmid no. 18656) (Takahashi and Yamanaka 2006). We transferred each ORF individually into pMXs-gw by performing site-specific LR recombination using Gateway LR Clonase II enzyme mix kit (Thermo Fisher Scientific).

\section{Retrovirus production, cellular reprogramming, and treatment}

For retroviruses production, Platinum E cells were seeded into culture dishes $\left(1 \times 10^{5}\right.$ cells per square centimeter $) 1 \mathrm{~d}$ before transfection in DMEM supplemented with $10 \%$ FBS and $1 \%$ penicillin/streptomycin. Cells reached $\sim 80 \%$ confluency on the day of transfection. DNA plasmids were transfected into Platinum E cells using FuGENE 6 transfection reagent. Twenty-four hours after transfection, wild-type or aMHC-GFP TTFs were seeded into culture dishes or plates that were precoated with SureCoat (Cellutron) for $1 \mathrm{~h}$ at a density of $6 \times 10^{3}$ per square centimeter. Forty-eight hours after transfection, polybrene was added to viral medium that was filtered through a $0.45-\mu \mathrm{m}$ filter at a concentration of $8 \mu \mathrm{g} / \mathrm{mL}$. The mixture replaced the growth medium in the cell culture plate with TTFs. The viral infection was serially repeated twice. Twenty-four hours after the second infection, the viral medium was replaced with induction medium, composed of DMEM/199 (4:1), 10\% FBS, 5\% horse serum, 1\% penicillin/ streptomycin, $1 \%$ nonessential amino acids, $1 \%$ essential amino acids, $1 \% \mathrm{~B}-27,1 \%$ insulin-selenium-transferrin, $1 \%$ vitamin mixture, and $1 \%$ sodium pyruvate (Invitrogen). Beating cell analysis was performed as described by Zhou et al. (2015). Small molecule treatments throughout the reprogramming process used 10 $\mu \mathrm{M}$ Dex, $10 \mu \mathrm{M}$ Nab (Sigma), and 10 nM FK228 (Selleckchem).

\section{Luciferase assay}

Expression constructs (pBx-GATA4 and pMXs-ZNF281) and a reporter construct ( $\mathrm{p}-\alpha \mathrm{MHC}$-Luciferase) were transfected into 
HEK293T cells using Fugene HD (Promega) according to manufacturer's instructions. The total amount of DNA per well was kept constant by adding the corresponding amount of expression vector without a cDNA insert. Twenty hours after transfection, cell extracts were assayed for luciferase expression using the luciferase assay kit (Promega). Relative promoter activities were expressed as fluorescence relative units normalized to pBX-GFP expression in the cell.

Flow cytometry

For flow cytometry, cells were trypsinized and fixed with fixation buffer (BD Bioscience) for 15 min on ice. Fixed cells were washed three times with Perm/Wash buffer (BD Bioscience). Washed cells were incubated with mouse monoclonal anti-cTnT antibody (Thermo Scientific, MA5-12960) at 1:200 dilution and rabbit anti-GFP antibody (Thermo Scientific, A-11122) at 1:200 dilution in Perm/Wash buffer for $1 \mathrm{~h}$ on ice. Cells then were washed three times with Perm/Wash buffer followed by incubation with donkey anti-mouse Alexa fluor 647 (Invitrogen, A-31571) at 1:200 and goat anti-rabbit Alexa fluor 488 (Invitrogen, A-11008) at 1:200. Cells were washed with Perm/Wash buffer and then analyzed using FACS Caliber (BD Sciences) and FlowJo software.

\section{Immunocytochemistry}

For immunocytochemistry, cells were fixed in $4 \%$ paraformaldehyde for $15 \mathrm{~min}$ and permeabilized with $0.1 \%$ Triton X-100 at room temperature. Cells were washed three times with PBS followed by blocking with $10 \%$ goat serum for $1 \mathrm{~h}$. Cells then were incubated with mouse monoclonal anti-cTnT antibody (Thermo Scientific, MA5-12960) at 1:500 dilutions and rabbit anti-GFP antibody (Thermo Scientific, A-11122) at 1:500 dilutions in 5\% goat serum for $1 \mathrm{~h}$. After three washes with PBS, cells then were incubated with donkey anti-mouse Alexa fluor 647 (Invitrogen, A31571) at 1:500 and goat anti-rabbit Alexa fluor 488 (Invitrogen, A-11008) at 1:500. Image acquisition was done on an INCell Analyzer 6000 (GE, Inc.) using a Nikon $4 \times$ objective. Samples were imaged using 488 and 561 laser lines for aMHC-GFP and cTnT, respectively. Four fields were used to capture the entire well. Image processing was performed in GE Developer Toolbox (version 1.9.3). The acquired images were segmented on the aMHC-GFP and cTnT channels separately. We then calculated the total area (square micrometers) and total signal intensity (arbitrary units) of each probe within their respective masks. Results for both channels were imported into the GeneData Analyzer module (version 13, GeneData, Inc.) and normalized against the robust median of the test population. From the normalized data, $Z$-scores were calculated as described previously (Wu et al. 2008).

\section{Quantitative mRNA measurement}

Total RNA was extracted using TRIzol (Invitrogen) according to the vender's protocol. RNAs were retrotranscribed to cDNA using iScript Supermix (Bio-Rad). qPCR was performed using KAPA SYBR Fast (Kapa Biosystems). mRNA levels were normalized by comparison with Gapdh mRNA. Primer sequences are listed in Supplemental Table S3.

\section{Immunoprecipitation and Western blot analysis}

Flag-epitope tag ZNF281 fusion proteins were coexpressed with myc-epitope tag fusion GATA4, HAND2, MEF2C, or TBX5 in HEK293 cells for $48 \mathrm{~h}$. Cell lysates were incubated overnight with $1 \mu \mathrm{g}$ of mouse monoclonal anti-Flag antibody (Sigma,
F3165). The cell lysates were pulled down using magnetic Protein G Dynabeads (Invitrogen), and then the Flag-ZNF281 was eluted using $0.5 \mathrm{mg} / \mathrm{mL}$ free Flag peptide (Sigma). The final elution and the input obtained before the immunoprecipitation were analyzed by SDS-PAGE Western blot using a mouse monoclonal anti-Myc antibody (Novex, R950-25) or mouse monoclonal anti-Flag antibody (Sigma, F3165).

\section{RNA-seq and $G O$ analysis}

For RNA-seq, total RNA was extracted from TTFs transduced with the indicated retroviruses using TRIzol (Invitrogen) according to the vender's protocol. Illumina RNA-seq was performed by the University of Texas Southwestern Microarray Core Facility. RNA-seq and transcriptome analysis were performed as described in Abad et al. (2017). Briefly, quality assessment of the RNA-seq data was done using NGS-QC-Toolkit. Reads with $>30 \%$ nucleotides with Phred quality scores $<20$ were removed from further analysis. Quality-filtered reads were then aligned to the mouse reference genome GRCm $38(\mathrm{mml})$ using the Hisat (version 2.0.0) aligner using default settings. Aligned reads were counted using featureCounts (version 1.4.6) per gene ID. Differential gene expression analysis was done using the R package edgeR (version 3.8.6). For each comparison, genes were required to have $10 \mathrm{cpm}$ (counts per million) in at least three samples to be considered as expressed. They were used for normalization factor calculation. Gene differential expression analysis was done using the GLM approach following edgeR official documentation. Cutoff values of fold change $>2$ and FDR $<0.01$ were then used to select for differentially expressed genes between sample group comparisons. The DAVID gene functional annotation and classification tool was used to annotate the list of differentially expressed genes. GO enrichment analysis was performed to determine molecular and biological functional categories. The enrichment plots were performed using GSEA software. All RNA-seq data have been deposited in the Sequence Read Archive (SRA) with the accession numbers listed in Supplemental Table S4.

\section{ChIP-seq, GO, and pathway analysis}

For ChIP-seq, TTFs were transduced with the indicated retroviruses. Two days after retroviral transduction, TTFs were crosslinked with $1 \%$ formaldehyde in PBS for $30 \mathrm{~min}$ and neutralized by the addition of glycine to a final concentration of $0.125 \mathrm{M}$ for 5 min. TTFs were harvested and washed with cold PBS, and then ChIP was performed using anti-GATA4 antibody (Santa Cruz Biotechnology, sc-1237), anti-ZNF281 antibody (Abcam, ab101218), and ChIP-IT Express ChIP kits (Active Motif) according to the vender's protocol. Subsequent library construction and massive parallel sequencing were performed at the University of Texas Southwestern Genomics and Microarray Core Facility. For ChIP-seq analysis, raw reads were mapped to GRCm38 (mm10) using Bowtie2 (version 2.2.8). An average of $\sim 50$ million uniquely mapped (single end) reads was obtained per sample. Peak calling was performed using MACS2 (version 2.1.0). Resulting peak files were processed using bedTools (version 2.26) and deepTools (Ramirez et al. 2016) to generate coverage heat maps and obtain overlapping regions. The GREAT kits (McLean et al. 2010) were used for GO and pathway analysis. All ChIP-seq data have been deposited in the SRA with the accession numbers listed in Supplemental Table S4.

\section{Quantification and statistical analysis}

All data are presented as mean with SEM and have $n=3$ per group (except Supplemental Fig. S4, with $n=6$ per group). $P$-values were 
calculated with either unpaired/two-way $t$-test or one-way ANOVA. Statistical analyses were run using the GraphPad Prism 7 software package. $P<0.05$ was considered significant in all cases after corrections were made for multiple pairwise comparisons.

\section{Acknowledgments}

We thank Jose Cabrera for assistance with graphics. This work was supported by grants from the National Institutes of Health (NIH; HL130253, HL-077439, DK-099653, and AR-067294), the Foundation Leducq Networks of Excellence (14CVD04 to E.N. O.), the Cancer Prevention and Research Institute of Texas (RP150596 to M.S.K.), and the Robert A. Welch Foundation (grant 1-0025 to E.N.O.). H.H. is supported by a Uehara Memorial Foundation Post-doctoral Fellowship and a Kanae Foreign Study Grant. M.G.M. is a Pew Latin American Fellow in the Biomedical Sciences, supported by the Pew Charitable Trusts. H.Z. was supported by a predoctoral fellowship (14PRE20030030) from the American Heart Association. We are also grateful for the support provided to the High-Throughput Screening Core Facility (B.A.P. and H.N.) by the Simmons Cancer Center Support Grant (P30 CA142543-06) and the NIH-sponsored S10 grant (1S10OD018 005-01 to B.A.P.) awarded for the INCell Analyzer 6000.

\section{References}

Abad M, Hashimoto H, Zhou H, Morales MG, Chen B, BasselDuby R, Olson EN. 2017. Notch inhibition enhances cardiac reprogramming by increasing MEF2C transcriptional activity. Stem Cell Reports 8: 548-560.

Ang YS, Rivas RN, Ribeiro AJ, Srivas R, Rivera J, Stone NR, Pratt K, Mohamed TM, Fu JD, Spencer CI, et al. 2016. Disease model of GATA4 mutation reveals transcription factor cooperativity in human cardiogenesis. Cell 167: 1734-1749.e22.

Aurora AB, Olson EN. 2014. Immune modulation of stem cells and regeneration. Cell Stem Cell 15: 14-25.

Aurora AB, Porrello ER, Tan W, Mahmoud AI, Hill JA, BasselDuby R, Sadek HA, Olson EN. 2014. Macrophages are required for neonatal heart regeneration. I Clin Invest 124: 1382-1392.

Cahill TJ, Choudhury RP, Riley PR. 2017. Heart regeneration and repair after myocardial infarction: translational opportunities for novel therapeutics. Nat Rev Drug Discov doi: 10.1038/ nrd.2017.106.

Cirillo LA, Lin FR, Cuesta I, Friedman D, Jarnik M, Zaret KS. 2002. Opening of compacted chromatin by early developmental transcription factors HNF3 (FoxA) and GATA-4. Mol Cell 9: $279-289$.

Fidalgo M, Shekar PC, Ang YS, Fujiwara Y, Orkin SH, Wang J. 2011. Zfp281 functions as a transcriptional repressor for pluripotency of mouse embryonic stem cells. Stem Cells 29: 1705-1716.

Fidalgo M, Faiola F, Pereira CF, Ding J, Saunders A, Gingold J, Schaniel C, Lemischka IR, Silva JC, Wang J. 2012. Zfp281 mediates Nanog autorepression through recruitment of the NuRD complex and inhibits somatic cell reprogramming. Proc Natl Acad Sci 109: 16202-16207.

Fidalgo M, Huang X, Guallar D, Sanchez-Priego C, Valdes VI, Saunders A, Ding J, Wu WS, Clavel C, Wang J. 2016. Zfp281 coordinates opposing functions of Tet 1 and Tet 2 in pluripotent states. Cell Stem Cell 19: 355-369.

Firulli BA, Milliar H, Toolan KP, Harkin J, Fuchs RK, Robling AG, Firulli AB. 2017. Defective Hand1 phosphoregulation uncov- ers essential roles for Hand 1 in limb morphogenesis. Development 144: 2480-2489.

Fu JD, Stone NR, Liu L, Spencer CI, Qian L, Hayashi Y, DelgadoOlguin P, Ding S, Bruneau BG, Srivastava D. 2013. Direct reprogramming of human fibroblasts toward a cardiomyocytelike state. Stem Cell Reports 1: 235-247.

Gomez-Del Arco P, Perdiguero E, Yunes-Leites PS, Acin-Perez R, Zeini M, Garcia-Gomez A, Sreenivasan K, Jimenez-Alcazar M, Segales J, Lopez-Maderuelo D, et al. 2016. The chromatin remodeling complex Chd4/NuRD controls striated muscle identity and metabolic homeostasis. Cell Metab 23: 881-892.

Hahn S, Hermeking H. 2014. ZNF281/ZBP-99: a new player in epithelial-mesenchymal transition, stemness, and cancer. J Mol Med 92: 571-581.

Han Z, Yi P, Li X, Olson EN. 2006. Hand, an evolutionarily conserved bHLH transcription factor required for Drosophila cardiogenesis and hematopoiesis. Development 133: 11751182.

Huang GN, Thatcher JE, McAnally J, Kong Y, Qi X, Tan W, DiMaio JM, Amatruda JF, Gerard RD, Hill JA, et al. 2012. C/ EBP transcription factors mediate epicardial activation during heart development and injury. Science 338: 1599-1603.

Ieda M, Fu JD, Delgado-Olguin P, Vedantham V, Hayashi Y, Bruneau BG, Srivastava D. 2010. Direct reprogramming of fibroblasts into functional cardiomyocytes by defined factors. Cell 142: 375-386.

Ifkovits JL, Addis RC, Epstein JA, Gearhart JD. 2014. Inhibition of TGF $\beta$ signaling increases direct conversion of fibroblasts to induced cardiomyocytes. PLoS One 9: e89678.

Kojima H, Ieda M. 2017. Discovery and progress of direct cardiac reprogramming. Cell Mol Life Sci 74: 2203-2215.

Lai SL, Marin-Juez R, Moura PL, Kuenne C, Lai JKH, Tsedeke AT, Guenther S, Looso M, Stainier DY. 2017. Reciprocal analyses in zebrafish and medaka reveal that harnessing the immune response promotes cardiac regeneration. Elife 6: e25605.

Lee J, Sayed N, Hunter A, Au KF, Wong WH, Mocarski ES, Pera RR, Yakubov E, Cooke JP. 2012. Activation of innate immunity is required for efficient nuclear reprogramming. Cell 151: $547-558$.

Luna-Zurita L, Stirnimann CU, Glatt S, Kaynak BL, Thomas S, Baudin F, Samee MA, He D, Small EM, Mileikovsky M, et al. 2016. Complex interdependence regulates heterotypic transcription factor distribution and coordinates cardiogenesis. Cell 164: 999-1014.

McLean CY, Bristor D, Hiller M, Clarke SL, Schaar BT, Lowe CB, Wenger AM, Bejerano G. 2010. GREAT improves functional interpretation of cis-regulatory regions. Nat Biotechnol 28: 495-501.

Mohamed TM, Stone NR, Berry EC, Radzinsky E, Huang Y, Pratt K, Ang YS, Yu P, Wang H, Tang S, et al. 2017. Chemical enhancement of in vitro and in vivo direct cardiac reprogramming. Circulation 135: 978-995.

Mouse ENCODE Consortium, Stamatoyannopoulos JA, Snyder M, Hardison R, Ren B, Gingeras T, Gilbert DM, Groudine M, Bender M, Kaul R, et al. 2012. An encyclopedia of mouse DNA elements (Mouse ENCODE). Genome Biol 13: 418.

Muraoka N, Yamakawa H, Miyamoto K, Sadahiro T, Umei T, Isomi $\mathrm{M}$, Nakashima $\mathrm{H}$, Akiyama $\mathrm{M}$, Wada $\mathrm{R}$, Inagawa $\mathrm{K}$, et al. 2014. MiR-133 promotes cardiac reprogramming by directly repressing Snail and silencing fibroblast signatures. EMBO J 33: 1565-1581.

Nam YJ, Song K, Luo X, Daniel E, Lambeth K, West K, Hill JA, DiMaio JM, Baker LA, Bassel-Duby R, et al. 2013. 
Reprogramming of human fibroblasts toward a cardiac fate. Proc Natl Acad Sci 110: 5588-5593.

Qian L, Huang Y, Spencer CI, Foley A, Vedantham V, Liu L, Conway SJ, Fu JD, Srivastava D. 2012. In vivo reprogramming of murine cardiac fibroblasts into induced cardiomyocytes. $\mathrm{Na}$ ture 485: 593-598.

Ramirez F, Ryan DP, Gruning B, Bhardwaj V, Kilpert F, Richter AS, Heyne S, Dundar F, Manke T. 2016. deepTools2: a next generation web server for deep-sequencing data analysis. Nucleic Acids Res 44: W160-W165.

Ramirez-Carrozzi VR, Nazarian AA, Li CC, Gore SL, Sridharan R, Imbalzano AN, Smale ST. 2006. Selective and antagonistic functions of SWI/SNF and Mi-2 $\beta$ nucleosome remodeling complexes during an inflammatory response. Genes Dev 20: 282-296.

Song K, Nam YJ, Luo X, Qi X, Tan W, Huang GN, Acharya A, Smith CL, Tallquist MD, Neilson EG, et al. 2012. Heart repair by reprogramming non-myocytes with cardiac transcription factors. Nature 485: 599-604.

Soufi A, Garcia MF, Jaroszewicz A, Osman N, Pellegrini M, Zaret KS. 2015. Pioneer transcription factors target partial DNA motifs on nucleosomes to initiate reprogramming. Cell 161: 555-568.

Srivastava D, DeWitt N. 2016. In vivo cellular reprogramming: the next generation. Cell 166: 1386-1396.

Srivastava D, Thomas T, Lin Q, Kirby ML, Brown D, Olson EN. 1997. Regulation of cardiac mesodermal and neural crest development by the bHLH transcription factor, dHAND. Nat Genet 16: 154-160.

Takahashi K, Yamanaka S. 2006. Induction of pluripotent stem cells from mouse embryonic and adult fibroblast cultures by defined factors. Cell 126: 663-676.

Tallquist MD, Molkentin JD. 2017. Redefining the identity of cardiac fibroblasts. Nat Rev Cardiol 14: 484-491.
Vaseghi H, Liu J, Qian L. 2017. Molecular barriers to direct cardiac reprogramming. Protein Cell doi: 10.1007/s13238017-0402-X.

Wang L, Liu Z, Yin C, Asfour H, Chen O, Li Y, Bursac N, Liu J, Qian L. 2015. Stoichiometry of Gata4, Mef2c, and Tbx5 influences the efficiency and quality of induced cardiac myocyte reprogramming. Circ Res 116: 237-244.

Wu Z, Liu D, Sui Y. 2008. Quantitative assessment of hit detection and confirmation in single and duplicate high-throughput screenings. J Biomol Screen 13: 159-167.

Xin M, Olson EN, Bassel-Duby R. 2013. Mending broken hearts: cardiac development as a basis for adult heart regeneration and repair. Nat Rev Mol Cell Biol 14: 529-541.

Yamakawa $\mathrm{H}$, Muraoka N, Miyamoto K, Sadahiro T, Isomi $M$, Haginiwa S, Kojima H, Umei T, Akiyama M, Kuishi Y, et al. 2015. Fibroblast growth factors and vascular endothelial growth factor promote cardiac reprogramming under defined conditions. Stem Cell Reports 5: 1128-1142.

Yang SY, Baxter EM, Van Doren M. 2012. Phf7 controls male sex determination in the Drosophila germline. Dev Cell 22: 1041-1051.

Zhao Y, Londono P, Cao Y, Sharpe EJ, Proenza C, O'Rourke R, Jones KL, Jeong MY, Walker LA, Buttrick PM, et al. 2015. High-efficiency reprogramming of fibroblasts into cardiomyocytes requires suppression of pro-fibrotic signalling. Nat Commun 6: 8243.

Zhou H, Dickson ME, Kim MS, Bassel-Duby R, Olson EN. 2015. Akt1/protein kinase B enhances transcriptional reprogramming of fibroblasts to functional cardiomyocytes. Proc Nat1 Acad Sci 112: 11864-11869.

Zhou Y, Wang L, Vaseghi HR, Liu Z, Lu R, Alimohamadi S, Yin C, Fu JD, Wang GG, Liu J, et al. 2016. Bmil is a key epigenetic barrier to direct cardiac reprogramming. Cell Stem Cell 18: 382-395. 


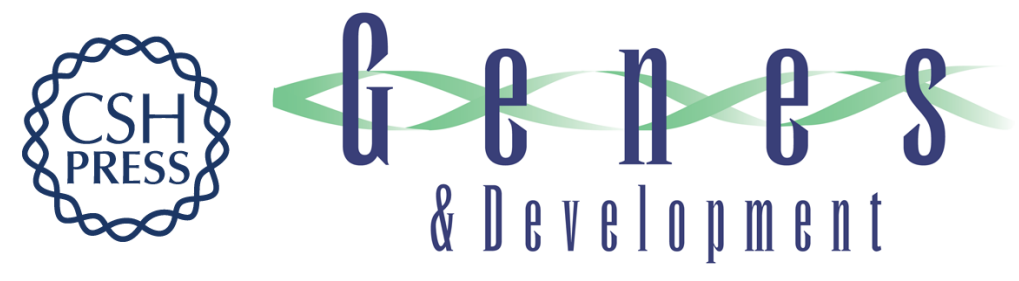

\section{ZNF281 enhances cardiac reprogramming by modulating cardiac and inflammatory gene expression}

Huanyu Zhou, Maria Gabriela Morales, Hisayuki Hashimoto, et al.

Genes Dev. 2017, 31:

Access the most recent version at doi:10.1101/gad.305482.117

Supplemental http://genesdev.cshlp.org/content/suppl/2017/10/05/31.17.1770.DC1
Material

References This article cites 44 articles, 10 of which can be accessed free at:

http://genesdev.cshlp.org/content/31/17/1770.full.html\#ref-list-1

Creative This article is distributed exclusively by Cold Spring Harbor Laboratory Press for the first

Commons

License

Email Alerting

Service six months after the full-issue publication date (see

http://genesdev.cshlp.org/site/misc/terms.xhtml). After six months, it is available under a Creative Commons License (Attribution-NonCommercial 4.0 International), as described at http://creativecommons.org/licenses/by-nc/4.0/.

Receive free email alerts when new articles cite this article - sign up in the box at the top right corner of the article or click here.

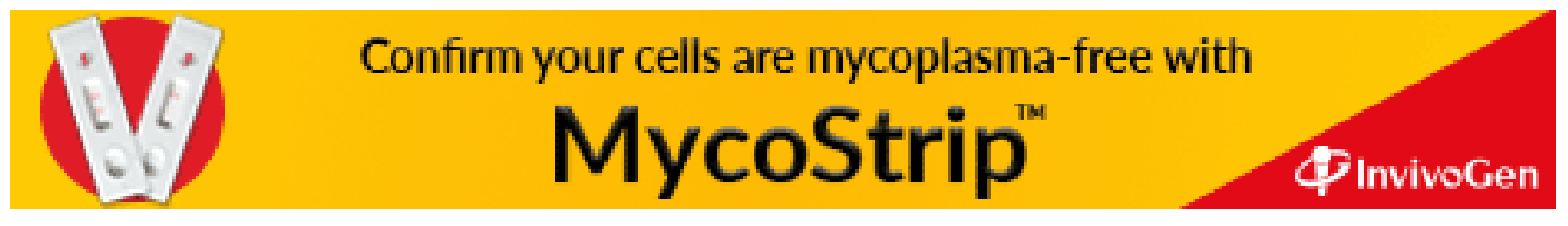

